\title{
Air Gap Diffusion Distillation: A New Method for Solution Separation Technology
}

\author{
Xuan Zhang, Shiming Xu, Ping Wang*, Lin Xu, Lei Li, Lele Chen \\ Key Laboratory of Ocean Energy Utilization and Energy Conservation of Ministry of Education, \\ School of Energy and Power Engineering, Dalian University of Technology
}

\begin{abstract}
Membrane distillation is a distillation technology that has emerged in the last 50 years.Compared with the traditional thermal distillation, membrane distillation has the advantages of compact structure, simple device and easy operation, so many membrane distillation technologies have been developed up to now.Recently, a novel air gap diffusion distillation (AGDD) technique has been proposed.This paper mainly introduces the research progress of air gap membrane distillation, solarenergy distillation and humidification and dehumidification technology in recent years.
\end{abstract}

Keywords: Air Gap Diffusion Distillation; Air Gap Membrane Distillation; Solar Distillation; Humidifying and dehumidifying

\section{Introduction}

Membrane distillation technology is a distillation technology put forward in 1960s[1], which relies on the temperature difference between hot and cold feed liquids separated by microporous hydrophobic membranes to drive the hot side to generate water vapor and diffuse to the cold side to complete the condensation distillation process. This is also the working principle of the first direct contact distillation designed. However, the heat conduction loss from the hot side to the cold side is very large. To solve this problem, air gap membrane distillation has been developed. The so-called air gap type membrane distillation is based on direct contact membrane distillation, filling air gap between hydrophobic microporous membrane and condensation plate wall to to reduce the conduction heat loss from the hot side to the cold side, but due to the existence of air gaps, the thermal resistance of the water vapor transmission process is increased. It is necessary to increase the temperature difference between the hot side and the cold side to ensure the driving force required for water vapor diffusion. With the deepening of membrane distillation technology research, and put forward the gas sweep and vacuum membrane distillation technology. The advantage of these two membrane distillation methods lies in the use of inert gas blowing water vapor diffused into the air gap is removed by sweeping or vacuum pump suction to enhance the pressure difference between the air gaps, thereby enhancing water large membrane flux can be obtained by the flow of vapor. However, both methods require additional condensing devices to cool the extracted steam. Condensation, increased equipment volume and investment cost. To sum up, air gap membrane distillation technology and direct contact membrane distillation technology are compared has better thermal efficiency, does not need an additional condensing device compared with the air sweeping type and the vacuum type membrane distillation technology, so that the current air sweeping type and the vacuum type membrane distillation technology. The research work of membrane distillation mainly focuses on air gap membrane distillation. In addition, many related and cross-application technologies are constantly being mined. This makes the application of membrane distillation technology not only limited to the field of seawater desalination, but also active in heavy metal wastewater treatment agents. Purification and other fields. This paper introduces the air gap membrane distillation technology, solar

Copyright (C) 2020 Xuan Zhang et al.

doi:10.18282/pef.v9i1.847

This is an open-access article distributed under the terms of the Creative Commons Attribution Non-Commercial License (http://creativecommons. org/licenses/by-nc/4.0/), which permits unrestricted non-commercial use, distribution, and reproduction in any medium, provided the original work is properly cited. 
distillation technology and humidification and dehumidification distillation in recent years. Development status, and combined with the three distillation methods, the innovation and practicability of the new distillation mode of air gap diffusion distillation are added. Discussion shows that air gap diffusion distillation has a good development prospect.

\section{Air gap membrane distillation technology}

At present, membrane distillation technology has many problems, such as low membrane flux, serious membrane pollution, expensive membrane preparation, and low cost in heat transfer and distillation. There are some uncertain factors in the mass transfer process, which makes the air gap membrane distillation currently in the experimental research stage and limits the membrane distillation. Practical application of technology. Scholars from the flow mode and flow type, membrane material membrane module and air gap type, heat source type and should be to carry out research work on air gap membrane distillation technology from the perspective of fields, as shown in Figure 1.

\section{Air gap membrane distillation}

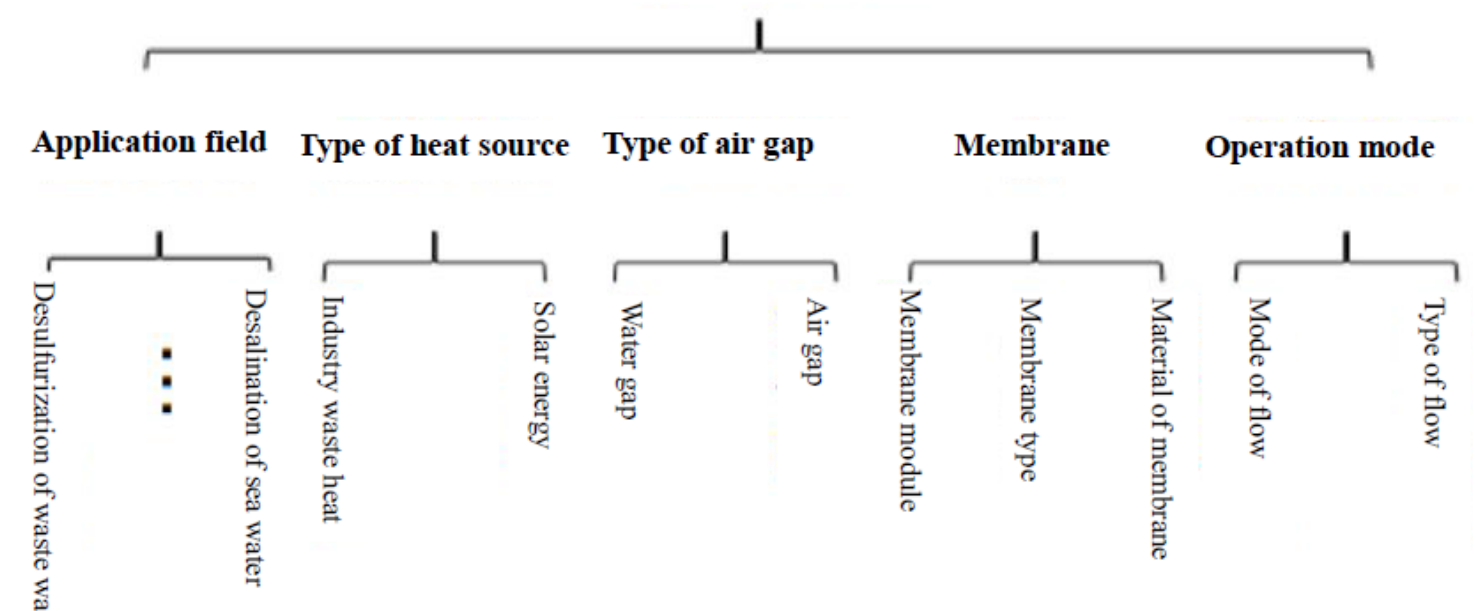

Figure 1. The framework for the development of air gap membrane distillation.

\subsection{Flow mode and flow type}

The most important factors affecting membrane distillation are temperature polarization and concentration polarization in the membrane distillation process. Yang Lan et al. ${ }^{[2]}$ adopted direct contact membrane distillation to investigate microporous hydrophobic membranes with different material structures. The results showed that the appropriate operation was controlled. Temperature and liquid flow rate can weaken the phenomenon of temperature polarization and obtain greater membrane flux and thermal efficiency, but there is high demand on the membrane's resistance to temperature and mechanical properties. Wang Hongtao et al. ${ }^{[3]}$ have adopted aeration-enhanced air gap membrane distillation to weaken the boundary layer by increasing the disturbance degree of feed liquid. The influence further obtains the higher membrane flux. Yang Xiaohong etc. ${ }^{[4]}$ have strong air blowing to tubular hollow fiber membrane module and flat membrane module respectively. The simulation of the chemical process and the experimental study of the aeration mode (continuous aeration or intermittent aeration) show that for hollow for fiber membrane modules, the gas-liquid flow ratio at about 0.5 can enhance the membrane flux better and indirect aeration is more effective than continuous aeration, while aeration is not ideal for its flat membrane modules. Li Hua et al. ${ }^{[5]}$ analyzed the process of aeration enhanced air gap membrane distillation from a simulation perspective and discussed the mechanism of aeration enhanced mass transfer, providing valuable reference methods for studying the process of membrane distillation enhanced mass transfer with multiple influencing factors.

\subsection{Film and film materials}

Microporous hydrophobic membrane is the core component of membrane distillation technology. The performance parameters of the membrane itself have a great influence on the overall performance of the system. Therefore since then, scholars at home and abroad have made extensive research on membrane materials. Sabeur Khemakhem et al. ${ }^{[6]}$ adopt alkyl triethoxysilane graft method two new ceramic hydrophobic porous membranes were prepared and air gap 
membrane distillation experiments were carried out to measure the water vapor transmission rate of the membranes. Results indicated that the new membrane has broad application prospects in the field of membrane distillation. Arcadio Sotto et $a l^{[7]}$ have studied polyethersulfone (PES) influence of sublayer on properties of polyamide (PA), asymmetric polymer sublayer was synthesized by diffusion induced phase separation (DIPS) $\mathrm{TiO}_{2 \_}$nanoparticles were added to enhance the membrane flux. A. Hemmat ${ }^{[8]}$ have explored the effects of different salt additives $\left(\mathrm{LiCl}_{1} \mathrm{CaCl}, \mathrm{CaCO}_{3}\right)$ on the preparation of polyvinylidene fluoride,-hexafluoropropylene, (PVDF-HFP) nanofiber membranes for air gap membrane distillation. The results show that the contact angle of PVDF-HFP film decreases with the increase of salt content, and the porosity of the film increases with the addition of salt, of which $\mathrm{LiCl}$ is the most obvious, reaching $89 \%$.

\subsection{Membrane module and air gap type}

Scholars have devoted a lot of research to the structural design of membrane modules. Current mainstream membrane modules include: hollow fibers Membrane modules such as type I, sleeve type and plate frame type etc. Wang Peng et al. ${ }^{[9]}$ have DESIgned and successfully manufactured lotus through special desi Porous PVDF hollow fiber (MBF) membrane with lotus-shaped geometry has shown superior stability in vapor permeation flux and salt inhibition under stable operating conditions in continuous direct contact membrane distillation (DCMD) experiments. Li Boyi, ${ }^{[10]}$ a hollow fiber membrane module added with a heat-insulating tubular partition net and arranged in a spiral winding structure and self-made by people and introduced The performance of air gap membrane distillation seawater desalination process was studied. Zhang Chunyao, ${ }^{[11]}$ and others use polypropylene-based hollow fiber membranes and a new type of energy recovery membrane module for polypropylene hollow fiber heat exchange tubes was developed to investigate the effect of membrane module structural parameters on total mass transfer coefficient. It is believed that increasing the membrane pore size can effectively improve the total mass transfer coefficient, increase the membrane flux and water production ratio, and increase the module length. Degree will reduce membrane flux. Liu Zhiyu, ${ }^{[12]}$ et al. aim at uneven distribution of air gap width of tubular hollow fiber air gap membrane distillation module and is difficult to control, a sleeve-type hollow with uniform air gap width is designed by using capillary copper tubes as heat exchange tubes fiber membrane air gap membrane distillation module. And the air gap membrane distillation process is coupled with vacuum membrane distillation technology to reduce the performance of non-condensable gases the adverse effects of. Kai Xu, ${ }^{[13]}$ and others used simulation software to construct a three-dimensional model for the plate-and-frame air gap membrane distillation module, revealing a new type. The intuitive parameter distribution law inside the module lays a foundation for further optimization of the internal structure of the new plate-and-frame air gap membrane module. Some scholars have improved the air gap structure and proposed a water gap membrane distillation method. Cheng Lan, ${ }^{[14]}$ Equal Pair Air Gap Membrane Distillation Performance. On the basis of improvement, the quality and heat transfer process of the existing membrane distillation module are analyzed, and the theoretical water gap formula is obtained. The performance of membrane distillation will be better. In fact, although the water gap membrane distillation can greatly improve the ratio of water production to heat production in low grade. The effect of water gap type on membrane flux is not much or even lower than that of air gap type membrane distillation. atia e. khalifa ${ }^{[15]}$ etc. slave operations air gap membrane distillation and water gap membrane distillation are discussed in terms of parameters and design parameters. It is believed that the increase of water gap thickness has great influence on distillation process. The influence is smaller than the increase of air gap thickness.

\section{Solar distillation technology}

Solar distillation technology is commonly used in the fields of seawater desalinization and seawater desalinization. There are two structural forms ${ }^{[16]}$, one is the distillation process of seawater phase change driven by heat energy generated by solar energy. The other is electrodialysis driven by solar power generation.

\subsection{Improvement of structure}

Solar distillation has various structures, and the ceiling type solar distiller commonly used at home and abroad in the early days is a passive type of distillation. Distillation has the advantages of simple structure, easy manufacture, low cost, etc. However, due to the great influence of the thermal inertia in the distiller, it leads to its water yield and thermal efficiency are relatively low and gradually replaced by other forms. At present, the common structures of solar 
distillation are inclined type and tray type, type, condensing type, multi-effect distillation and trough type condensing multi-effect distillation etc. In 1988 Zheng Weiqing ${ }^{[17]}$ and others developed inclined core distillation. Compared with the traditional ceiling distiller, the inclined core distiller is a solar desalination device with higher thermal efficiency. One of the devices. Hiroshi Tanaka, ${ }^{[18]}$ and others use flat reflectors to improve tilted core solar distillation. Li Qiang et al. ${ }^{[19]}$ carried out experiments and simulations to improve the structure of tilting solar distillation, and a regenerative tilting core solar distillation device was developed according to the design study, it is believed that reducing the feed water flow and making the inclination angle close to the local latitude will increase the water yield and evaporation efficiency, and at the same time the introduction of heat storage material greatly increases the nighttime water production and improves the overall water production effect. Zhang Xiaoyan et al. ${ }^{[20]}$ have explored the differences. Under the condition of overlapping plates, the relationship between the operating temperature, energy consumption and water yield of the distiller is analyzed and compared with single-stage, second-stage and third-stage distillation. Unit energy consumption and water production rate of the boiler. Research shows that the higher the operating temperature, the more the number of overlapping plates can be increased, and the total water production and transportation have an approximate linear relationship between income. Ju Yukun, ${ }^{[21]}$ and others have carried out solar disk distillation technology by means of dynamic strengthening. The experimental research shows that although power intensification can increase the water yield of the device, it also increases the energy consumption additionally. Chang Zehui ${ }^{[22]}$ et al. put forward the concept of concentrating solar seawater desalination system and carried out the economic analysis of concentrating solar seawater desalination system assessment. Yang Junwei, ${ }^{[23]}$ and others have developed single-stage and three-stage condensing direct-heat humidifying and dehumidifying solar seawater desalination devices and installed them in pairs. The internal heat and mass transfer process is analyzed, the simulation calculation model of the experimental device is established, and the calculation results and the experimental results are compared. The comparison shows that the simulation model can better predict the water production performance of the device.

\subsection{Performance improvement of heat collection system}

Zhang $\mathrm{Da},{ }^{[24]}$ has made their own solar simulator and used single tube heat pipe vacuum tube as the research object, and added on the back of the device. The installation of reflector can greatly improve the daily average efficiency. At the same time, the fin heat pipe is proposed as a cylindrical single-tube collector. Compared with flat-plate singletube collector, it is believed that the structure of water heater with cylindrical heat absorber is more advantageous, but reflection is not discussed. Influence of mirror material and other heat pipe structures except cylindrical heat pipe on performance. Shufu ${ }^{[25]}$ and others proposed a new type of heat pipe vacuum tube collector using graphite material as heat conducting medium. Experiments show that the heat collecting efficiency can reach $65.8 \%$, which is very important for the development of new type of solar energy. The collector has important reference value.

\section{Humidification and dehumidification distillation technology}

With the deepening of research, researchers from various countries have proposed many improvement measures for humidifying and dehumidifying distillation technology. Humidification and dehumidification distillation methods can be classified as shown in Figure $2^{[26]}$. Mainly from the driving mode and heat and mass transfer mode to humidifying and dehumidifying technology To discuss the technique. 


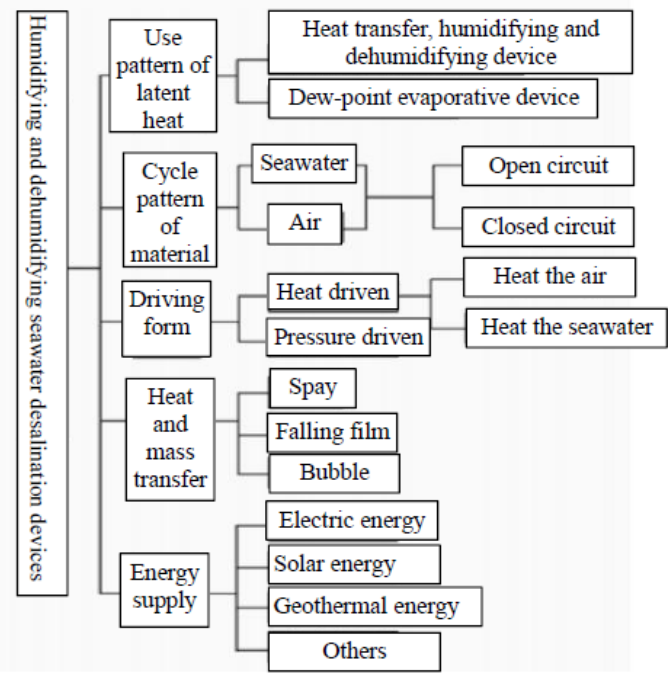

Figure 2. The classification of humidifying and dehumidifying seawater desalination devices.

\subsection{Driving Form}

The driving forms of humidifying and dehumidifying distillation technology can be thermal driving and pressure $\operatorname{driving}^{[27]}$.

Energy index. The results show that for the heating seawater type humidifying and dehumidifying device, increasing the energy consumption of the dehumidifier can obtain a higher water production ratio, and to a certain extent, increasing the mass flow rate can also improve the water production ratio. Saeed Dehghani, ${ }^{[28]}$ and others have studied the use of heat pump assistance.

Direct contact with dehumidification device and adding various parameters to theoretical model to evaluate system performance.

\subsection{Heat and Mass Transfer Mode}

Humidification and dehumidification distillation structures are various, and Lin $\mathrm{Zhe}^{[29]}$ and others have designed a humidification and dehumidification solar sea with overlapping structures.

Compared with the traditional humidifying and dehumidifying device, the water desalination system has the characteristics of compact structure. Meanwhile, light generated by the device is utilized water sprays cooling air, reducing the use of condensers. Tao Jun, ${ }^{[30]}$ and others have established multiple levels based on mass balance and heat balance. The setting of blowing volume provides the basis. penghuigao ${ }^{[31]}$ and others have designed a new type of bubble tower for coal mine wastewater treatment. Humidification and dehumidification equipment and related experiments. Ali I shehata ${ }^{[32]}$ etc. optimize the humidifying and dehumidifying distillation system by using hot material spraying and ultrasonic technology. Experiments have proved that, ultrasonic humidification is beneficial to water production and can reduce the cost of water production.

\section{Air gap diffusion distillation technology}

\subsection{Proposal of air gap diffusion technology}

Air Gap Diffusion Distillation Technology (AGDD: air gap diffusion distillation is a new distillation technology based on the air gap membrane distillation technology. Xu Shiming, ${ }^{[33]}$ and others have proposed an air gap diffusion distillation model and built an air gap diffusion distillation experiment table. The structural principle is shown in Figure 3. Inspection feed the effects of operating parameters such as temperature, feed flow rate, air gap thickness and device height on the ratio of water production to water production are compared with those of other structural parameters. The simulation results are compared to verify the correctness of the model and the feasibility of the scheme. 


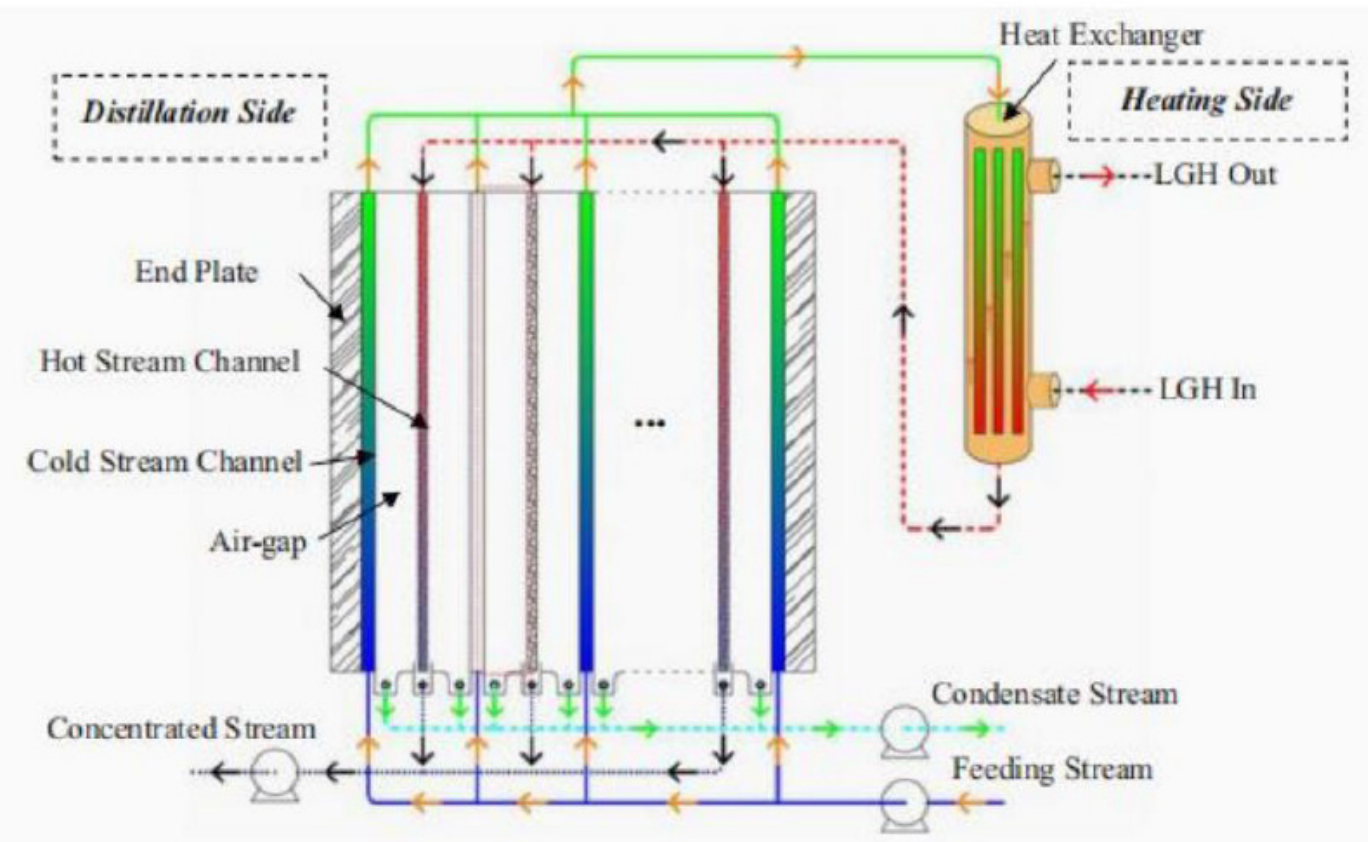

Figure 3. The schematic diagram of structure of air gap diffusion distillation system.

Yu Bingchen, ${ }^{[34]}$ and others have studied the heat and mass transfer characteristics of air gap diffusion seawater desalination device by establishing physical and mathematical models to explore the influence of structural parameters on water yield and thermal efficiency. Zhang Shuping et al. ${ }^{[35]}$ the experimental test results were compared with the program simulation calculation results, and the effects of operation parameters, size and working liquid concentration on the seawater desalination performance of the device were analyzed.

\subsection{Application prospect of air gap diffusion distillation technology}

Various distillation technologies can not only be independently applied in the fields of seawater desalination, wastewater desulfurization, reagent purification, etc., but also be coupled with other technologies. Yuan Yang, ${ }^{[36]}$ and others propose an electrochemical cycle (charging-free TREC,TREC:) that uses low-grade heat to achieve thermal regeneration without additional electrical consumption. Thermally Regenerative Electrochemical Cycle Xu Shiming ${ }^{[37]}$, etc. have proposed a kind of technology to generate electricity by using low-grade heat energy to generate salt difference energy to drive, RED, solution concentration difference, heat, electricity and conversion technology. Its structural cycle is shown in Figure 4.

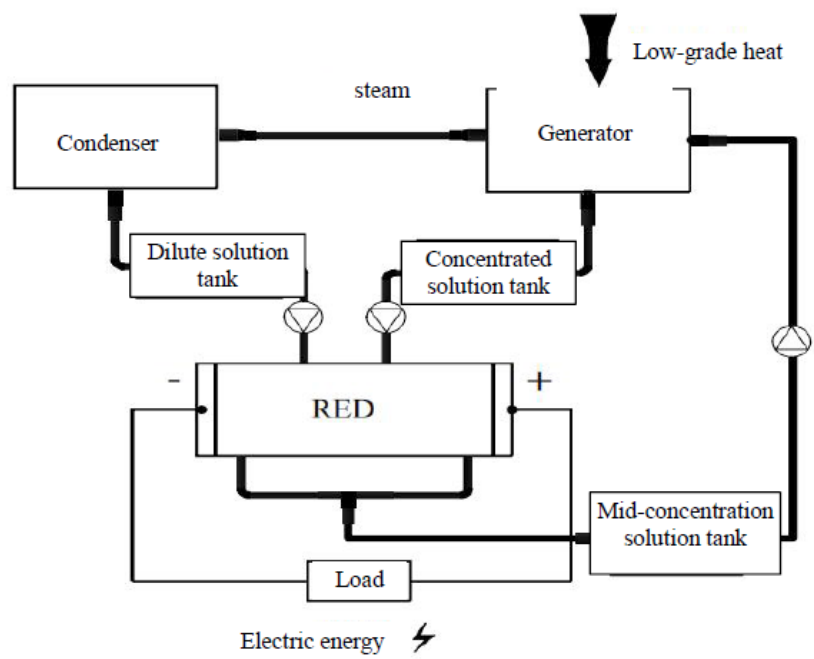

Figure 4. The basic cycle of solution concentration energy "thermo-electric" conversion.

The cycle can be divided into two parts: a solution separation device and a reverse electrodialysis device (RED). Long Rui, ${ }^{[38]}$ and others proposed a will hybrid membrane power generation system for converting low-grade heat energy into electric energy, i.e. realizing solution separation through membrane distillation technology to generate salt differential energy, and the salt difference can be used in the reverse electrodialysis device and the influence of 
performance on the device is systematically investigated. Hu Junyong ${ }^{[39]}$, et al. analyzed the influence of operating parameters and structural parameters on the system through theoretical models of construction, low temperature multieffect distillation, reverse electrodialysis power generation. Patricia Palenzuela, ${ }^{[40]}$ et al. analyzed the performance of RED-MED and Salt Gradient Heat Engine. It is believed that the influence of operating parameters on the overall system is more complex and some favorable operating conditions have been determined. In the 28 and efficiency systems, the flow rates of dilute solution $(0.01 \mathrm{M})$ and concentrated solution $(3.5 \mathrm{M})$ are $1 \mathrm{~cm} / \mathrm{s}$ and $0.5 \mathrm{~cm} / \mathrm{s}$ respectively. Under these conditions, the exergy efficiency can approach $7 \%$ and exergy efficiency can reach $31 \%$ for ideal films. B.OrtegaDelgado $^{[41]}$, et al. Exergy Analysis of Multi-effect Distillation, Reverse Electrodialysis Heat Engine. F. Giac Alone, ${ }^{[42]}$ et al. used experiments to verify the reverse electrodialysis heat engine model and evaluate the influence of current commercial membranes and future enhanced membranes on system efficiency. Giac Alone, ${ }^{[43]}$ and others have analyzed the thermodynamic characteristics and prospects of the new working medium for closed-loop reverse electrodialysis, explored the thermodynamic performance of several unconventional solutions and their effects on the performance of closed-loop reverse electrodialysis, and correlated the data with the Pitzer model.

\section{Conclusion}

This paper introduces the research progress of air gap membrane distillation technology, solar distillation technology and humidification and dehumidification technology in recent years. Air gap type compared with solar energy technology and warming and dehumidification, membrane distillation technology has simple structure, convenient operation and higher thermal efficiency. However, the core component of this technology is hydrophobic microporous membrane structure, and the existence of the membrane will inevitably lead to the water vapor transmission process blocked, resulting in reduced water production. Although scholars have proposed membrane distillation technology from the aspects of membrane materials and device structure design, etc. Many improvement schemes, but due to the high price and easy fouling of the membrane itself, the investment cost and maintenance cost of the device are increased. It is difficult to realize commercial mass production. Scholars should continue to search for one or more kinds of height from the material selection and preparation process of the membrane. Cost-effective membrane making process to speed up the practical process of membrane distillation technology. The advantage of solar distillation technology lies in the use of solar energy for cleaning. Energy is the driving force to enhance the utilization value of energy. However, the disadvantage is that this method is greatly influenced by geographical location and climatic conditions. Research on heat storage materials and collectors has improved the thermal efficiency of solar distillation technology to some extent. Moisturizing and Dehumidifying Steam compared with solar distillation technology, distillation has the advantages of higher thermal efficiency and water yield, but humidification and dehumidification distillation and solar distillation the area required for energy distillation technology is relatively large, which increases the land cost and limits the development of these two technologies. Air gap membrane distillation, solar distillation and humidification and dehumidification distillation technologies still need to be improved. At present, a new distillation technology, i.e. air gap diffusion distillation technology. The technique has been proposed. AGDD Hydrophilic porous materials used in the device are not only cheap but also have large evaporation area, small thermal resistance and heat high efficiency; Meanwhile, the occupied area of the device is small, thus effectively reducing the cost. In addition, the technology can not only be used independently but also it can be coupled with other technologies and has a good application prospect.

\section{References}

1. Huan Guolan, Du Qiyun, Wang Wei. Research status of membrane distillation technology [J]. Journal of tianjin polytechnic university, 2009,28(4):12-18.

2. Yang Lan, Ding Zhongwei, Ma Runyu. Study on the Effect of Temperature Polarization on Membrane Distillation Process [J]. Membrane Science and Technology, 2004,24(03):4-9.

3. Wang Hongtao. Analysis of Cross-flow Vacuum Membrane Distillation Process and Study on Component Amplification Characteristics [D]. Tianjin: Tianjin University, 2012.

4. Hu Junhu, Wu Shunli, Yang Xiaohong, Tian Rui, Liu Changyou, Yan Wenzhi. Experimental Study on Steam 
Distillation of Aerated Membrane [J]. Membrane Science and Technology, 2019,39(5):105-111.

5. Li Hua, Pan Yanqiu, Yu Lu, He Demin. Numerical simulation of mass transfer of air-gap membrane distillation with forced aeration [J]. Journal of Chemical Engineering of Higher Education, 2019,33(04):824-831.

6. S. Khemakhem, R. Ben. Ama, Modification of Tunisian clay membrane surface by silane grafting: Application for desalination with air gap membrane distillation process, Colloids and Surfaces A: Physicochemical and Engineering Aspects, 2011, 387:79-85.

7. A. Sotto, A. Rashed, R. X. Zhang, A. Martínez, L. Braken, P. Luis, B. Van der Bruggen, Improved membrane structures for seawater desalination by studying the influence of sublayers, Desalination, 2012, 287:317-325.

8. A. Hemmata, S. M. Ghoreishia, J. Karimi Sabet, Effect of Salt Additives on the Fabrication of Poly (vinylidene fluoride-co-hexafluropropylene) (PVDF-HFP) Nanofibe Membranes for Air Gap Membrane Distillation, Procedia Materials Scienc, 2015,11:370-375.

9. P.Wang, Tai-Shung Chung, Design and fabrication of lotus-root-like multi-bore hollow fiber membrane for direct contact membrane distillation, Journal of Membrane Science, 2012, 421-422:361-374.

10. Li Boyi, Wang Jianyou, Wang Jihu, Liu Hongbin. New hollow fiber air gap membrane distillation for seawater desalination [J]. Journal of Chemical Engineering, 2015. 66(1):149-156.

11. Zhang Chunyao, Geng Hongxin, Lang Qingcheng, Li Peili, Wu Xiaoyan. Desalination Process of New Air Gap Membrane Distillation Module [J]. Journal of Chemical Engineering, 2015. 66(10):4000-4006.

12. Liu Zhiyu. Design and Experimental Study of Casing Air Gap Membrane Distillation Module [D]. Tianjin: tianjin polytechnic university, 2018. “

13. Kai Xu, Li Baoan. CFD Numerical Simulation of Flow Field in New Plate and Frame Air Gap Membrane Distillation Module [J]. Membrane Science and Technology, 2017, 37(2):88-95.

14. Cheng Lan. Comparison of Air-gap and Water-gap Membrane Distillation Performance and Study on Membrane Pollution [D]. Tianjin: Tianjin University, 2018.

15. Atia E. Khalifa, Water and air gap membrane distillation for water desalination: An experimental comparative study, Separation and Purification Technology, 2015, 141:276-284.

16. Deng Xin. Research Status and Prospect of Solar Distillation Seawater Desalination Technology [J]. Silicon Valley, 2010(12):38.

17. Zheng Weiqing, Huang Yuanfeng, Feng Luobin, Fang Hui. Experimental Study on Tilted Core Solar Distiller [J]. Water Treatment Technology, 1988, 14(03):20-23.

18. H.Tanaka,T.Nosoko,T.Nagata,Experimental study of basin-type,multiple-effect,diffusioncoupled solar Stil, Desalination, 2002, 150:131-144.

19. Zhang Xiaoyan, Zheng Hongfei, Zhang Lianying. Experimental Study on Multistage Stacked Disk Solar Distiller [J]. Water Treatment Technology, 2003, 29(04):233-235.

20. Ju Yukun. Experimental Study on Desalination of Brackish Water by Solar-powered Intensified Disc Distillation System [D]. Beijing: China University of Geosciences, 2010.

21. Chang Zehui; Research on Thermophysical Problems of Concentrated Solar Seawater Desalination System; D; Beijing; Beijing University of Technology; 2014.

22. Yang Junwei ;Performance Study of Condensing Direct Heat Humidification and Dehumidification Solar Seawater Desalination System ;D ;Beijing ;Beijing University of Technology ;2016. “

23. Zhang Da. Experimental Study on Improving Thermal Performance of Solar Collectors [D]. Hohhot: Inner Mongolia University of Technology, 2009.

24. Li Qiang. Tilting Solar Distiller Experiment and CFD Simulation Research [D]. Tianjin: Hebei University of Technology, 2015.

25. Shu C. Study on thermal characteristics of graphite filled heat pipe vacuum tube collector [D]. Wuhan: Huazhong University of Science and Technology, 2015.

26. Tao Jun, Gong Jianguo, Zeng Sheng, Shan Yan, Jin Tao. Research progress on humidification and dehumidification seawater desalination technology [J]. Chemical Progress, 2012, 31(7):1419-1424.

27. L. Dahiru, M. Antar, K. Atia, Z. Syed, Al-Sulaiman Fahad, Humidification-dehumidification desalination system 
operated by a heat Pump, Energy Conversion and Management, 2018, 161:128-140.

28. S. Dehghani, A. Date, A. Akbarzadeh, Performance analysis of a heat pump driven humidification dehumidification desalination system, Desalination, 2018, 445:95-104.

29. Lin Zhe, Wu Gang, Yang Yingjun. Steady-state Study on Stacked Humidification and Dehumidification Solar Seawater Desalination System [J]. Solar Energy, 2017(9):32-36.

30. Tao Jun. Study on multi-stage solar-wind bubbling humidification and dehumidification seawater desalination technology [D]. Hangzhou: Zhejiang University, 2013.

31. Penghui Gao, Meng Zhang, Yuji Du, Bo Cheng and Donghai Zhang. Study on bubble column humidification and dehumidification system for coal mine wastewater treatment, Water Science \& Technology, 2018, 9:1909-1919.

32. Ali I. Shehata, A.E. Kabeel,M.Mohamed, Khairat Dawood, Abo Elazma, Abdall M. Abdalla, Ahmed Mehannaa, Achievement of humidification and dehumidification desalination system by utilizing a hot water sprayer and ultrasound waves techniques, Energy Conversion and Management, 2019, 201:112-142.

33. Shiming Xu, Lin Xu, Xi Wu, Ping Wang, Dongxu Jin, Junyong Hu, Shuping Zhang, Qiang Leng, Debing Wu, Airgap diffusion distillation: Theory and experiment, Desalination, 2019, 467:64-78.

34. Yu Bingchen. Study on heat and mass transfer characteristics of air gap diffusion seawater desalination [D]. Dalian: Dalian University of Technology, 2018.

35. Zhang Shuping. Experimental Study on Air Gap Diffusion Distillation Seawater Desalination [D]. Dalian: Dalian University of Technology, 2019.

36. Y. Yang, S.W. Lee, H. Ghasemi, J. Loomis, X. Li, D. Kraemer, G. Zheng, Y. Cui,G. Chen, Charging-free electrochemical system for harvesting low-grade thermal energy, Proc. Natl. Acad. Sci. 2014, 111:17011-17016.

37. Xu Shiming, Wu Xi, Wu Debing, Liu Huan, Zhang Hongjun, He Qichen, Zhang Kai, Chen Shunquan, Chen Jing, From Absorption Refrigeration to Reverse Electrodialysis Power Generation, Solution Concentration Energy. Application of New Technology, [J]. Refrigeration Technology, ,2017, 37(2):8-13.

38. Rui Long, Baode Li, Zhichun Liu, Wei Liu. Hybrid membrane distillation-reverse electrodialysis electricity generation system to harvest low-grade thermal energy.Journal of Membrane Science, 2017, 525:107-115.

39. Hu J, Xu S, Wu D, Jin D, Wang P, Leng Q. Theoretical simulation and evaluation for the performance of the performance of the hybrid multi-effect distillation-reverse electrodialysis power generation system, Desalination, 2018, 443:172-183.

40. Patricia Palenzuela, Marina Micari, Bartolomé Ortega-Delgado, Francesco Giacalone, Guillermo Zaragoza, DiegoCésar Alarcón-Padilla, Andrea Cipollina, Alessandro Tamburini, Giorgio Micale. Performance Analysis of a REDMED Salinity Gradient Heat Engine, Energies, 2018, 3385, 1-23.

41. B.Ortega-Delgado, F.Giacalone, P.Catrini, A.Cipollina, A.Piacentino, A.Tamburini, G.Micale. Reverse electrodialysis heat engine with multi-effect distillation: Exergy analysis and perspectives, Energy Conversion and Management, 2019, 194:12-25.

42. F.Giacalone, F.Vassallo, L.Griffin, M.C.Ferrari, G.Micale, F.Scargiali, A.Tamburini, Thermolytic reverse electrodialysis heat engine:model development,intergration and performance analysis, Energy Conversion and Management, 2019, 189:1-13.

43. F.Giacalone, C.Olkis, G.Santori, A.Cipollina, S.Brandani, G.Micale. Novel solutions for closed-loop reverse electrodialysis: Thermodynamic characterisation and perspective analysis, Energy, 2019, 166:674-689. 